\title{
Microfiltration en flux tangentiel des lactosérums de fromagerie
}

\author{
par \\ M. PIOT*, J.-L. MAUBOIS*, P. SCHAEGIS**, R. VEYRE*** \\ et LUCCIONI****
}

\section{Ré s u m é}

Plusieurs types de membranes de microfiltration ont été testés sur du lactosérum de fromagerie. Le but recherché lors de la microfiltration était de réaliser la clarification et l'épuration bactérienne du lactosérum ainsi que de permettre le passage d'un maximum de protéines du lactosérum au travers de la membrane.

Les résultats montrent que toutes les membranes présentent un colmatage vis-à-vis des protéines dans les conditions étudiées. Il est cependant possible d'obtenir une perméation protéique supérieure à $80 \%$ avec des microfiltres en polycarbonate ayant une porosité de 0,4 micron. Les valeurs de perméation protéique sont plus faibles avec les autres membranes en polymères ou avec les membranes minérales. Une réduction décimale des micro-organismes égale à 5 dans le microfiltrat a été obtenue.

Cependant, aucune des membranes testées ne satisfaisait les critères de rétention bactérienne et de perméation protéique évoqués ci-dessus avec un débit de microfiltration au moins égal à celui observé avec les membranes d'ultrafiltration.

Mots clés : Microfiltration tangentielle - Lactosérum - Membranes polymères Membranes minérales.

\section{S u m m a r y}

Several types of microfiltration membranes have been tested with cheese whey. The objective of microfiltration is to realize

* I.N.R.A., Technologie Laitière, 65, rue de Saint-Brieuc - 35042 Rennes.

** D.M.F., 19, rue Marc-Seguin - 75018 Paris.

*** S.F.E.C., B.P. $33-84500$ Bollène.

**** R.P. Recherche - 69-200 Vénissieux. 
clarification and bacterial reject from the cheese whey and to allow maximum permeability of whey proteins through the membrane. The results show that all the membranes clog up more or less with regard to the permeation of whey proteins in the microfiltrate under experimental conditions. Nevertheless, it is possible to obtain protein permeation of more than $80 \%$ with 0,4 micron polycarbonate microfilters but the values are lower with the other polymeric membranes or mineral membranes. We established that the decimal reduction of microorganisms in microfiltrate could be equal to 5 when compared to the cheese whey. Unfortunately we did not find the ideal membrane which would simultaneously satisfy all the above parameters and which would present a flux at least, similar with the one obtained with ultrafiltration membranes.

Key words: Cross-flow microfiltration - Whey proteins - Polymeric membranes Mineral membranes.

\section{INTRODUCTION}

Au cours de la décennie qui s'est écoulée, il s'est créé dans la plupart des grands pays laitiers du monde, une véritable industrie du lactosérum. Autrefois considéré comme un déchet, ce produit dérivé des industries fromagère et caséinière, est maintenant une matière première de base à l'origine de toute une variété de produits transformés dont certains à haute valeur ajoutée. Tel est le cas des préparations à destination diététique ou thérapeutique dans lesquelles entrent les protéines de lactosérum.

En effet, grâce aux technologies à membrane, ultrafiltration notamment, les protéines de qualité nutritionnelle exceptionnelle (Roger, 1979) qui sont présentes dans le lactosérum, peuvent être purifiées et concentrées dans des proportions convenant à de vastes besoins exprimés par des industries d'aval. Mais, de nombreux débouchés se trouvent encore limités pour les concentrés de protéines de lactosérum (C.P.L.) par la présence dans le lactosérum initial de composants limitant des propriétés fonctionnelles souhaitées ou susceptibles de conduire à des défauts de goût comme la matière grasse résiduelle. Il en est de même des micro-organismes contaminants dont la destruction thermique entraîne une dénaturation dommageable de ces protéines.

La microfiltration du lactosérum a été étudiée dès 1976 par Lee et Merson dans le but d'améliorer les performances des équipements d'ultrafiltration. Ces auteurs avaient, en effet, observé qu'une microfiltration sur membrane Nuclépore de $0,4 \mu$, réalisée en flux perpendiculaire, « clarifiait » le lactosérum de cottage-cheese étudié, par rétention sur la membrane de la majeure partie des fines de caséine, 
de la matière grasse résiduelle et des micro-organismes. Le débit d'ultrafiltration du lactosérum ainsi prétraité était multiplié par 3. Plus récemment, Mérin et coll. (1983) ont indiqué avoir obtenu à l'aide de microfiltres Gelman (membrane $0,8 \mu$ ) fonctionnant en flux tangentiel des débits de microfiltration sur lactosérum doux, à $37^{\circ} \mathrm{C}$ se stabilisant à $251 \mathrm{~h}^{-1} \mathrm{~m}^{-2}$, une rétention totale de la matière grasse et une rétention ne dépassant pas 5 à $6 \%$ pour les matières azotées. Les membranes utilisées par ces auteurs avaient un spectre de porosité assez large puisque la réduction décimale observée était comprise entre 1 et 2 . De plus, la rétention des protéines vraies n'était pas précisée puisque l'estimation était faite globalement par détermination Kjeldahl.

Considérant que la mise en œuvre de la microfiltration en flux tangentiel sur membranes de porosité comprise entre 0,2 et $0,5 \mu$ ne devait pas a priori être fondamentalement différente de celle de l'ultrafiltration sur membrane de porosité 50 à 100 fois plus faible, nous nous sommes attachés depuis 1980 dans le cadre d'un contrat ANVAR* à étudier l'insertion des membranes de microfiltration disponibles dans des équipements d'ultrafiltration, les caractéristiques des produits traversant la membrane (microfiltrats) ou retenus et la faisabilité technologique de l'insertion d'une telle opération dans l'industrie du lactosérum.

\section{MATERIEL ET METHODES}

\section{Matériel}

Les différents équipements utilisés ainsi que les caractéristiques des lactosérums employés sont décrits en tête de chaque chapitre.

\section{Méthodes de dosage}

- la matière azotée totale (exprimée en $\mathrm{Nx6,38)}$ était dosée par la méthode Kjeldahl ;

- l'azote non protéique (NPN) était déterminé également par la méthode de Kjeldahl sur le filtrat obtenu après précipitation des protéines par l'acide trichloracétique à $12 \%$;

- $\beta$-lactoglobuline et $\alpha$-lactalbumine : ce dosage était effectué par chromatographie liquide haute performance sur une colonne type TSK $3000 \mathrm{SW}$ avec élution par un tampon TRIS 0,01 M-HCl pH 6,7 et détection à $280 \mathrm{~nm}$;

Contrat Anvar $n^{\circ} 651922$. 
- la flore totale était déterminée par incubation pendant $72 \mathrm{~h}$ à $30^{\circ} \mathrm{C}$ sur Plate Count Agar Difco ;

- la flore coliforme était déterminée par incubation pendant 18 à $24 \mathrm{~h}$ à $30^{\circ} \mathrm{C}$ sur Violet Red Bile Agar Difco.

\section{RESULTATS EXPERIMENTAUX}

Nous avons testé successivement des membranes en polycarbonate et en polyester fabriqués par Nuclépore et commercialisées en France par DMF, des membranes en polymères Rhône-Poulenc et des membranes minérales SFEC.

\section{Membranes Nuclépore}

Ces membranes sont obtenues par irradiation d'un film en polycarbonate ou en polyester dans un réacteur nucléaire, il résulte de ce traitement une porosité à la fois très précise et très homogène.

\section{1.a) Essais en laboratoire}

Dans un premier temps et compte tenu de la dimension moléculaire des constituants à séparer, nous avons testé des membranes de porosité comprise entre 0,015 et 0,1 micron. Les essais ont été réalisés dans une cellule Amicon de $400 \mathrm{ml}$ à $20^{\circ} \mathrm{C}$, sous une pression d'azote de 3 bars.

Le lactosérum issu de l'égouttage de fabrications de fromages à pâtes molles (Laiterie Bridel, L'hermitage) était filtré sur toile avant son utilisation pour les expérimentations; le $\mathrm{pH}$ était voisin de 6,4. Le lactosérum était agité dans la cellule par un barreau aimanté tournant.

Les résultats obtenus sont rassemblés dans les graphiques 1 et 2 .

On constate que les débits de perméation augmentent avec le diamètre des pores jusqu'à 0,08 micron $(800 \mathrm{~A})$ puis un léger colmatage apparaît à 0,1 micron $(1000 \mathrm{~A})$. La perméation protéique est maximale à 0,05 micron $(500 \mathrm{~A})$.

Cette première série d'essais montre bien, si besoin était, le peu d'efficience d'une microfiltration en flux perpendiculaire qui aboutit à des rétentions proches de celles observées en ultrafiltration avec des membranes de porosité 100 fois plus élevée.

\section{1.b) Essais sur installation pilote}

Une deuxième série de membranes en polycarbonate de porosité 0,2 et 0,4 micron (2000 et $4000 \mathrm{~A}$ ) a été étudiée sur une installation pilote SRTI. 


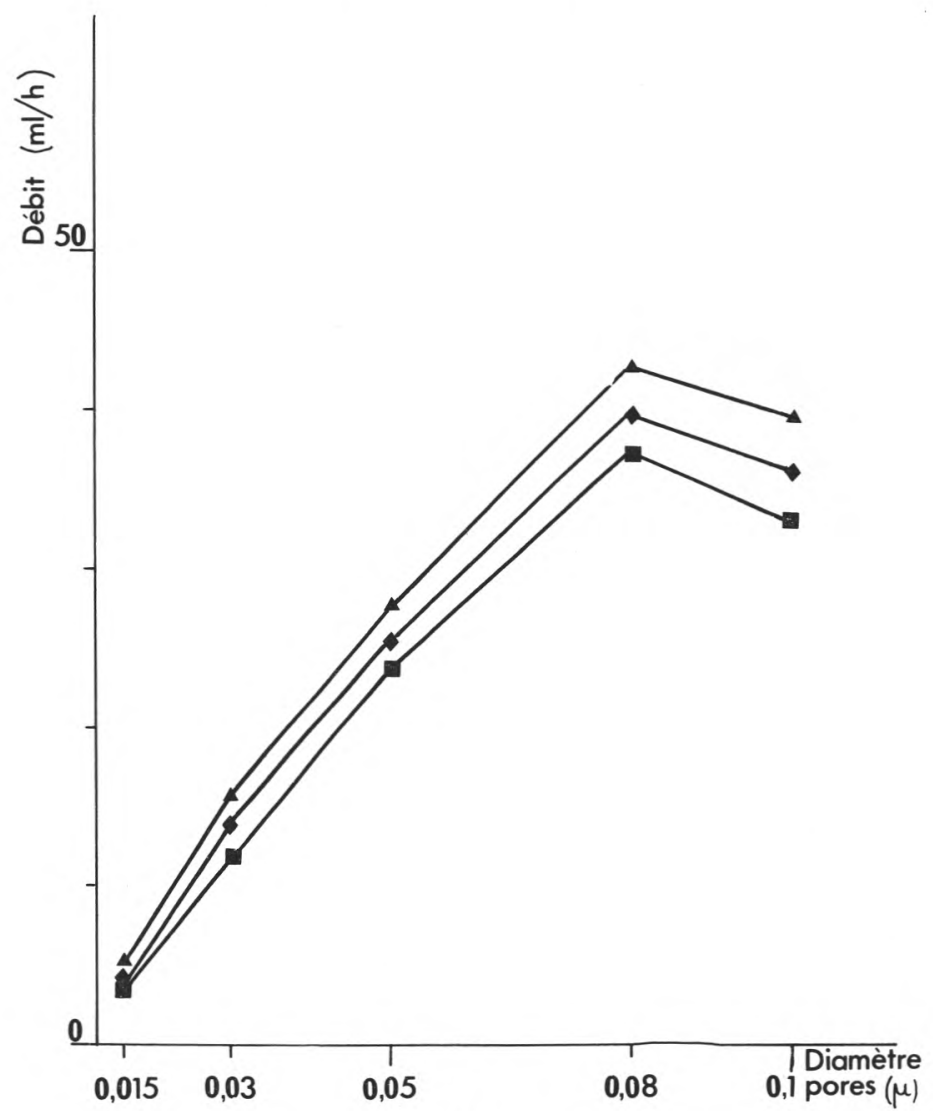

fig. 1

Débit de microfiltration après $1 \mathrm{~h}(\boldsymbol{\Delta}), 2 \mathrm{~h}(\bullet)$ et $3 \mathrm{~h}(\mathbf{E})$ pour différents diamètres de pores. Membranes Nuclépore, cellule 400 Amicon.

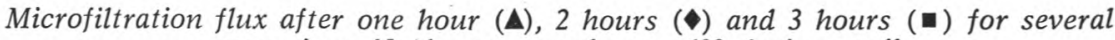
pore sizes. Nuclepore membrane, 400 Amicon cell.

Les conditions opératoires étaient les suivantes :

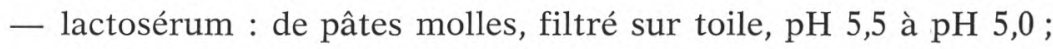

- pression : entrée 2 bars, sortie 0,5 bar (p. moyenne : 1,25 bar) ;

- température : $20^{\circ} \mathrm{C}$;

— vitesse de balayage : $4 \mathrm{~m} / \mathrm{s}$.

Les graphiques 3 et 4 représentent une sélection des meilleurs résultats, beaucoup d'essais ayant abouti à des fissures dans les membranes, voire même à des déchirures en raison des turbulences du 


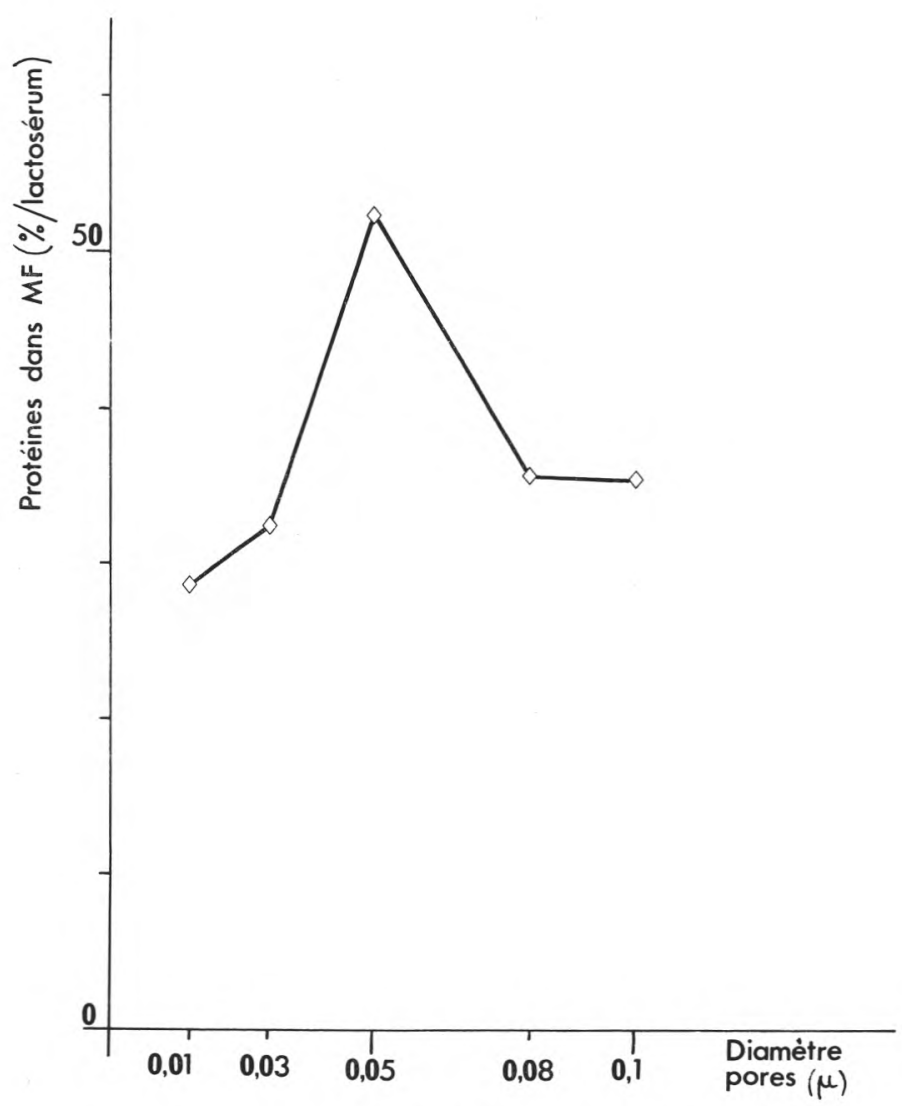

fig. 2

Pourcentage, par rapport au lactosérum, de protéines exprimées en (N. total NPN) 6,38 dans le microfiltrat pour différents diamètres de pores. Membranes Nuclépore, cellule 400 Amicon.

Proportion of whey proteins passing through the membrane for several pore sizes. Nuclepore membrane, 400 Amicon cell.

liquide circulant à grande vitesse (les membranes ont une épaisseur de 10 microns seulement).

Les membranes de porosité 0,4 micron présentent un débit de perméation plus élevé que les membranes 0,2 micron mais le colmatage est plus rapide pour les premières que pour les deuxièmes (après $4 \mathrm{~h}$ de fonctionnement les débits sont $56 \mathrm{l} / \mathrm{h} . \mathrm{m}^{2}$ bar pour 0,4 micron et $50 \mathrm{l} / \mathrm{h} \cdot \mathrm{m}^{2}$ bar pour 0,2 micron). Le pourcentage de protéines du lactosérum dans le microfiltrat diminue progressivement 


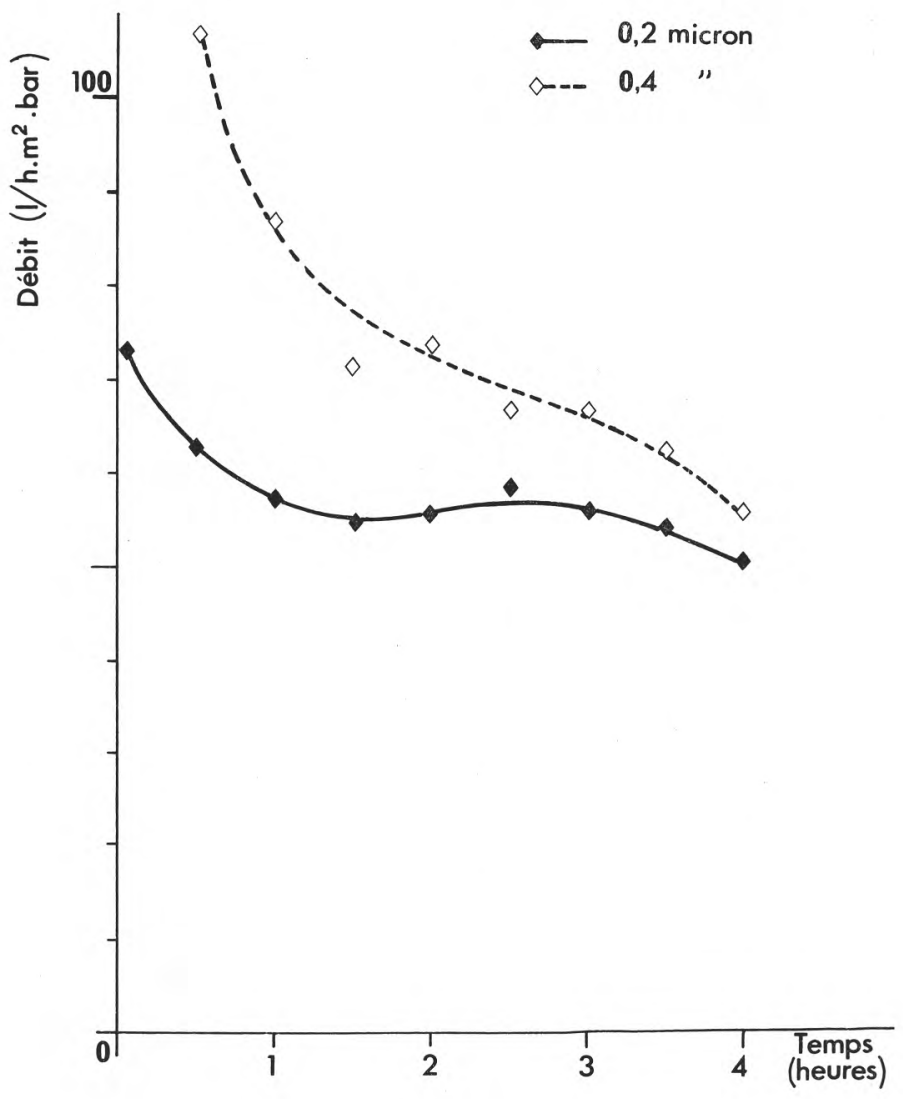

fig. 3

Débit de microfiltration obtenu sur membranes Nuclépore $0,2 \mu(\bullet)$ et $0,4 \mu(\diamond)$ en fonction du temps.

Microfiltration flux observed with $0,2 \mu(\diamond)$ and $0,4 \mu(\diamond)$ Nuclepore membrane.

au cours du temps et représente de 97 à $79 \%$ pour la membrane 0,4 micron et de 76 à $66 \%$ pour la membrane 0,2 micron. La membrane 0,4 micron est donc la plus performante de cette série de membranes.

1.c) Membranes Nucléopore polyester 0,4 micron renforcées

Ce nouveau type expérimental de membrane renforcée a été monté dans un module plan de type UFP 10 Rhône-Poulenc. 


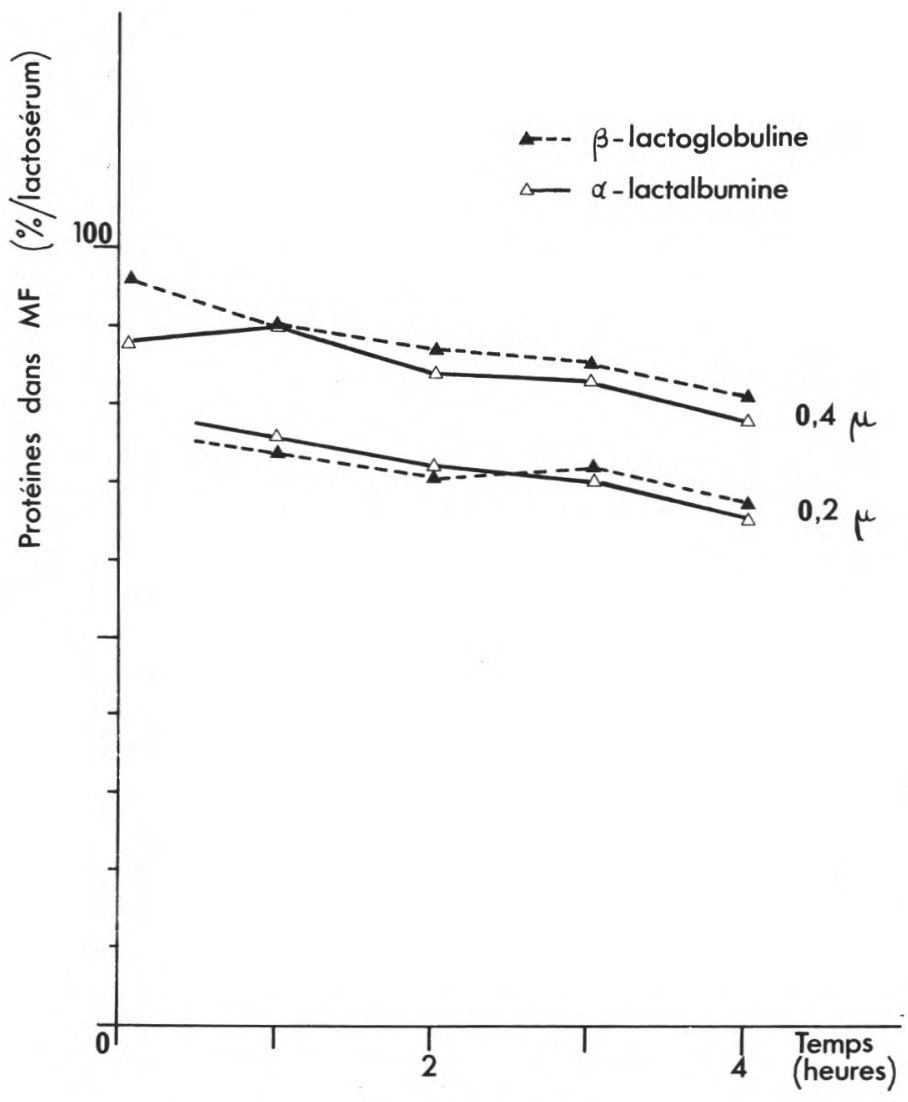

fig. 4

Teneur en $\beta$-lactoglobuline $(\mathbf{\Delta})$ et $\alpha$-lactalbumine $(\Delta)$ dans le microfiltrat par rapport au lactosérum en fonction du temps. Membranes Nuclépore $0,2 \mu$ et $0,4 \mu$.

$\beta$-lactoglobulin ( $\mathbf{\Delta})$ and $\alpha$-lactalbumin $(\Delta)$ contents in microfiltrate expressed in proportion of similar whey contents.

La comparaison des débits de microfiltration et des taux de perméabilité des deux principales protéines du lactosérum a été effectuée à $20^{\circ} \mathrm{C}$ et $50^{\circ} \mathrm{C}$ sur du lactosérum de fromagerie écrémé et pasteurisé à $72^{\circ} \mathrm{C}$ pendant 20 secondes, provenant d'un mélange de différentes fabrications, de $\mathrm{pH}$ voisin de 6,0, qui était fourni par les Ets Triballat à Noyal-sur-Vilaine. Le lactosérum était réensemencé à l'aide d'un mélange contenant des bactéries lactiques 


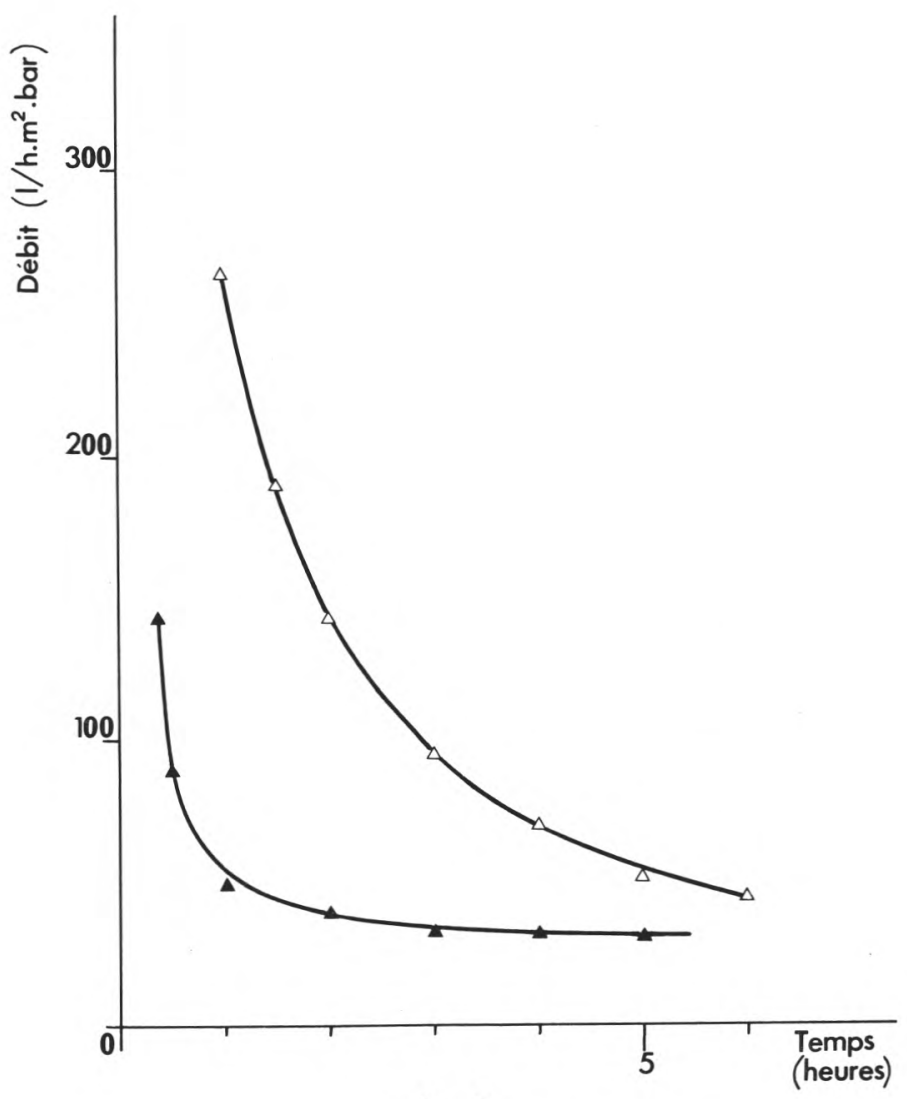

fig. 5

Débit de microfiltration à $20^{\circ} \mathrm{C}(\Delta)$ et $50^{\circ} \mathrm{C}(\triangle)$ en fonction du temps. Membranes Nuclépore $0,4 \mu$ renforcées.

Microfiltration flux at $20^{\circ} \mathrm{C}(\Delta)$ and $50^{\circ} \mathrm{C}(\triangle)$ observed with composite Nuclepore membranes.

et coliformes afin de suivre leur rétention au cours des essais et de contrôler la fiabilité des membranes.

Le colmatage est beaucoup plus progressif à $50^{\circ} \mathrm{C}$ qu'à $20^{\circ} \mathrm{C}$, en effet, le débit de microfiltration est pratiquement stabilisé après $2 \mathrm{~h}$ de fonctionnement à $20^{\circ} \mathrm{C}$ aux environs de $35 \mathrm{l} / \mathrm{h} . \mathrm{m}^{2}$ bar alors qu'à ce stade le débit à $50^{\circ} \mathrm{C}$ est beaucoup plus élevé $\left(140 \mathrm{l} / \mathrm{h} . \mathrm{m}^{2}\right.$ bar $)$ ensuite il diminue progressivement même après $6 \mathrm{~h}$ de fonctionnement tout en restant encore supérieur à la valeur mesurée à $20^{\circ} \mathrm{C}$ (graphique 5). 


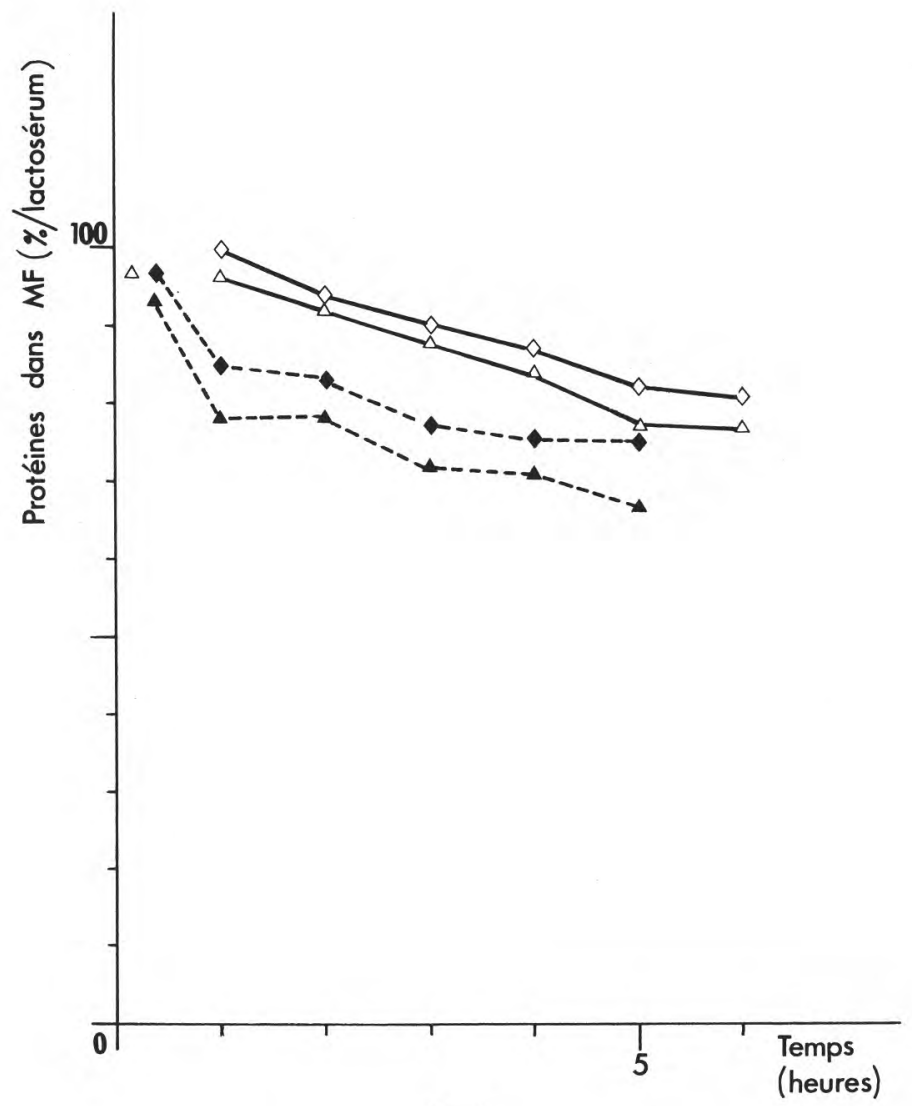

fig. 6

Pourcentage par rapport au lactosérum, de $\beta$-lactoglobuline à $20^{\circ} \mathrm{C}(\mathbf{\Delta})$ et $50^{\circ} \mathrm{C}$ $(\Delta)$ et d' $\alpha$-lactalbumine à $20^{\circ} \mathrm{C}(\diamond)$ et $50^{\circ} \mathrm{C}(\diamond)$ dans le microfiltrat en fonction du temps. Membranes Nuclépore 0,4 $\mu$ renforcées.

$\beta$-lactoglobulin and o-lactalbumin microfiltrate contents expressed in proportion of similar whey proteins contents at $20^{\circ} \mathrm{C}$ and $50^{\circ} \mathrm{C}$. Composite Nuclepore 0,4 $\mu$.

La perméabilité des protéines est également plus élevée à $50^{\circ} \mathrm{C}$ avec ce type de lactosérum, on note également (graphique 6) une rétention légèrement plus importante de la $\beta$-lactoglobuline par rapport à l'a-lactalbumine surtout à $20^{\circ} \mathrm{C}$.

On constate également une diminution de la teneur en matière grasse du microfiltrat ainsi qu'on peut le voir dans le tableau 1. Toutes les membranes Nuclépore testées satisfont largement nos exigences en matière de rétention des bactéries et de clarification. 


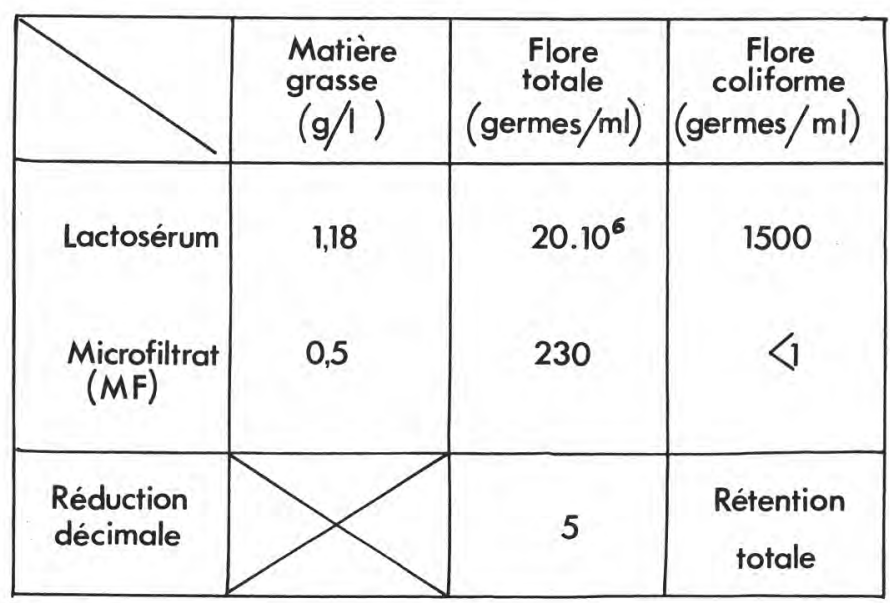

TABLEAU 1 - TABLE 1

Teneur en matière grasse et dénombrement bactérien du lactosérum et du microfiltrat obtenu sur membrane Nuclépore

Fat content and bacterial count of initial whey and of microfiltrate obtained from Nuclepore membrane

(cf. tab. 1), par contre leur défaut majeur serait la fragilité.

\section{Membranes Rhône-Poulenc}

Deux types de membranes planes en polymères (type A et type B) Rhône-Poulenc montées sur un module type UFP 10 ont été étudiés entre 20 et $25^{\circ} \mathrm{C}$ avec une vitesse de balayage de $2,5 \mathrm{~m} / \mathrm{s}$. Le même type de lactosérum que précédemment était utilisé.

Les débits de microfiltration mesurés sont les suivants (graphique 7) :

- type A : 68 1/h.m² bar;

- type B : inférieur à $40 \mathrm{l} / \mathrm{h} . \mathrm{m}^{2}$ bar après $1 \mathrm{~h}$ de fonctionnement.

Le taux de protéines dans le microfiltrat est représenté dans le graphique 8. La perméabilité est faible pour les membranes type A surtout pour la $\beta$-lactoglobuline (26\% lactosérum); quant aux autres membranes, les résultats sont légèrement meilleurs mais le colmatage est progressif, après $3 \mathrm{~h}$ de fonctionnement le passage de $\beta$-lactoglobuline est voisin de $45 \%$ et il représente $55 \%$ pour l' $\alpha$-lactalbumine. 


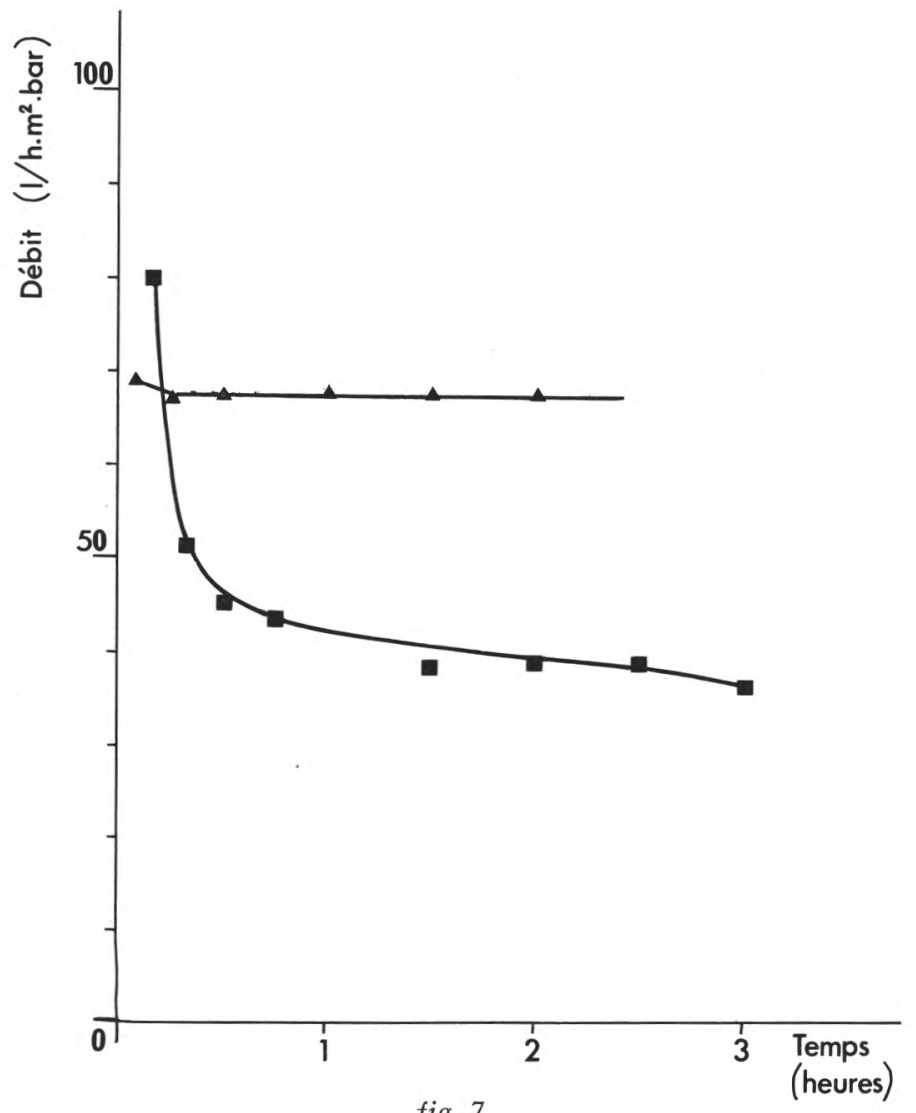

fig. 7

Débit de microfiltration en fonction du temps. Membranes Rhône-Poulenc

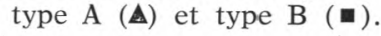

Microfiltration flux. Rhône-Poulenc membranes.

On peut faire deux remarques :

- les membranes type A ont un débit de microfiltration supérieur à celui des membranes type $\mathrm{B}$, mais également une rétention des protéines plus importante;

- comme on pouvait s'y attendre en raison de sa masse moléculaire et de son aptitude à se polymériser, la $\beta$-lactoglobuline est plus retenue par les membranes que l'a-lactalbumine.

D'autre part, ces membranes présentent également une porosité gênante des bactéries coliformes (réduction décimale de 3 pour le 


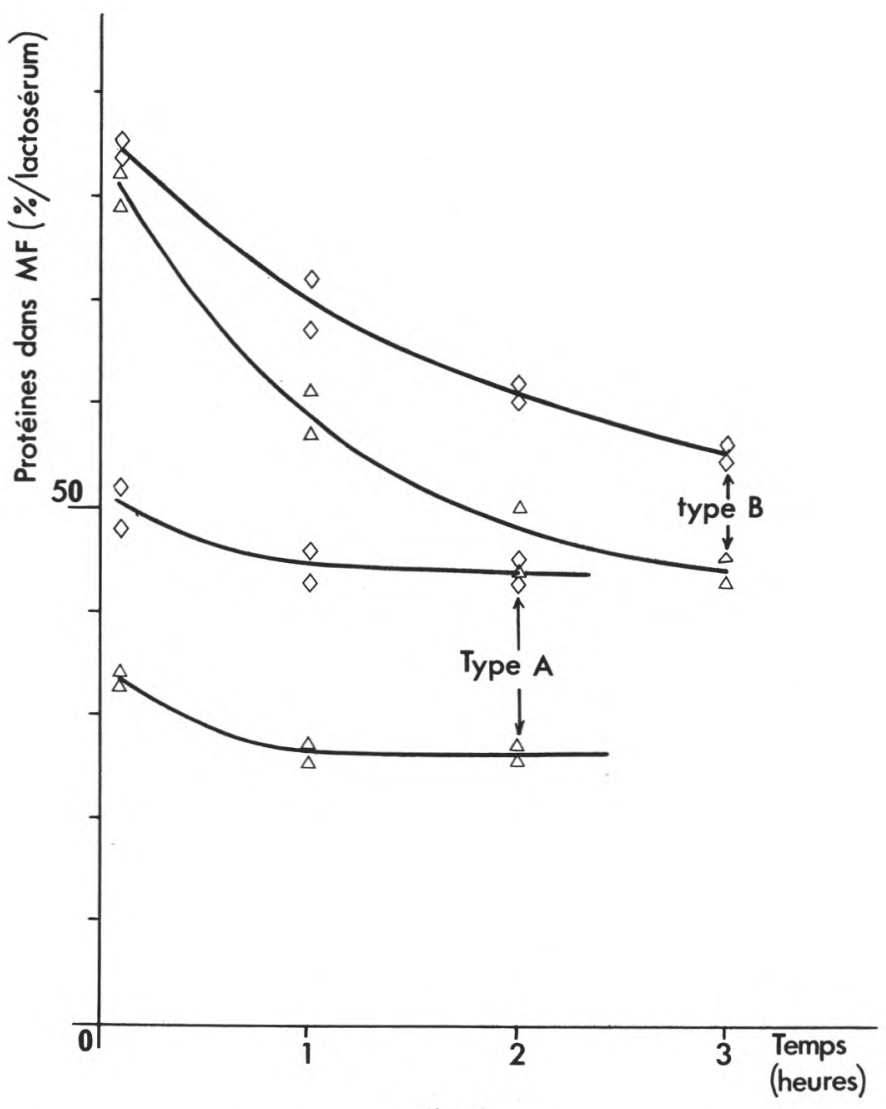

fig. 8

Pourcentage par rapport au lactosérum, de $\beta$-lactoglobuline $(\triangle)$ et d' $\alpha$-lactalbumine $(\diamond)$ dans le microfiltrat en fonction du temps. Membranes Rhône-Poulenc type A et type B.

Protein permeation of Rhône-Poulenc membranes.

type A, de 2 pour le type B) ce qui semble indiquer que la calibration des pores n'est pas assez homogène.

\section{Membranes Sfec}

La conception de ces membranes est très différente des précédentes. Ce sont des membranes tubulaires de diamètre intérieur $6 \mathrm{~mm}$ composées d'un support rigide en carbone recouvert sur sa face interne d'une couche d'oxyde de zirconium. La dimension exacte des pores ne nous est pas connue mais le classement des membranes 


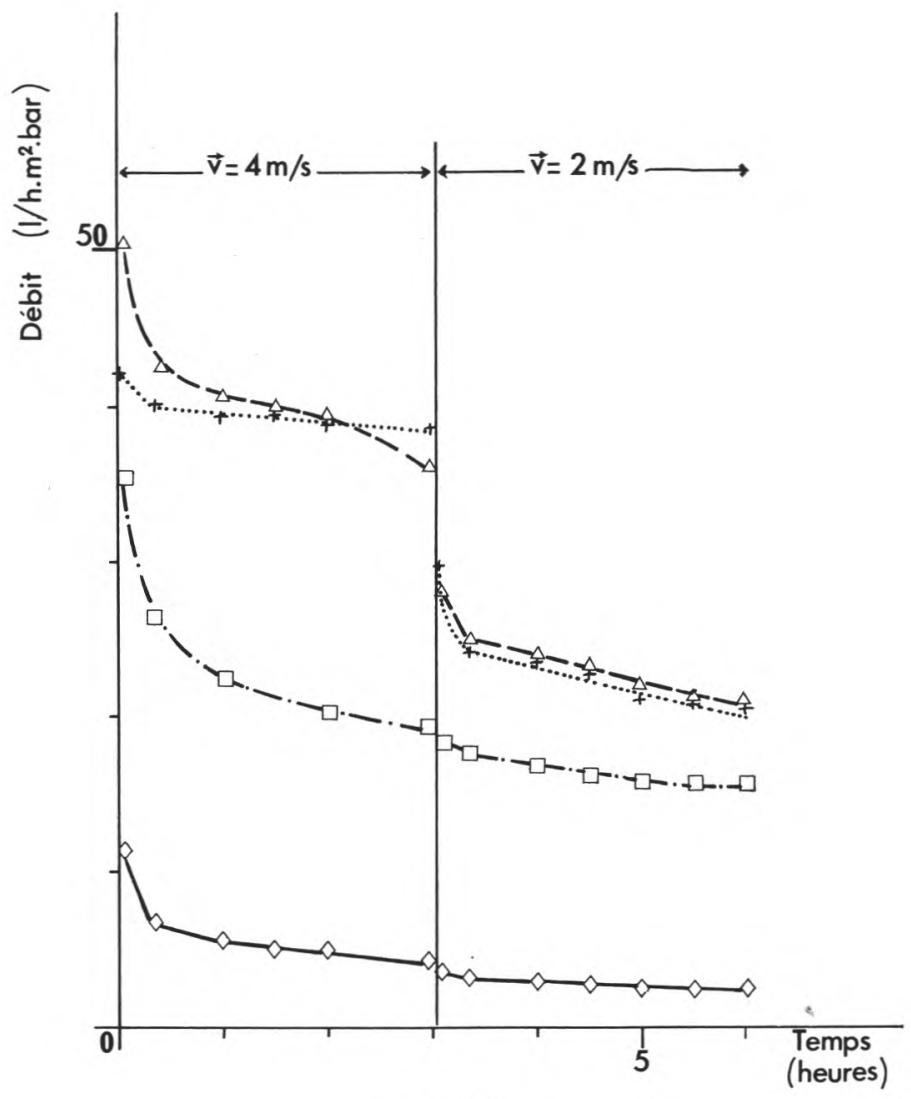

fig. 9

Débit de microfiltration en fonction du temps.

Membranes SFEC M $6(+)$, M $61000(\triangle), \operatorname{MCX}(\square)$ et support $(\diamond)$.

Microfiltration flux of SFEC membranes.

les unes par rapport aux autres peut être fait d'après le taux de rejet qu'elles présentent vis-à-vis d'un Dextran de masse moléculaire $2.10^{6}$.

Quatre types de membranes ont été testées sur une installation pilote avec du lactosérum à $20^{\circ} \mathrm{C}$ et $\mathrm{pH}$ sous une pression moyenne de 1,5 bar et pour des vitesses de balayage de $2 \mathrm{~m} / \mathrm{s}$ et $4 \mathrm{~m} / \mathrm{s}$. Les résultats correspondants apparaissent dans les graphiques 9, 10, 11 et 12 .

Le débit de microfiltration décroît rapidement lorsque le taux de rejet sur Dextran diminue sauf pour les deux membranes les plus 


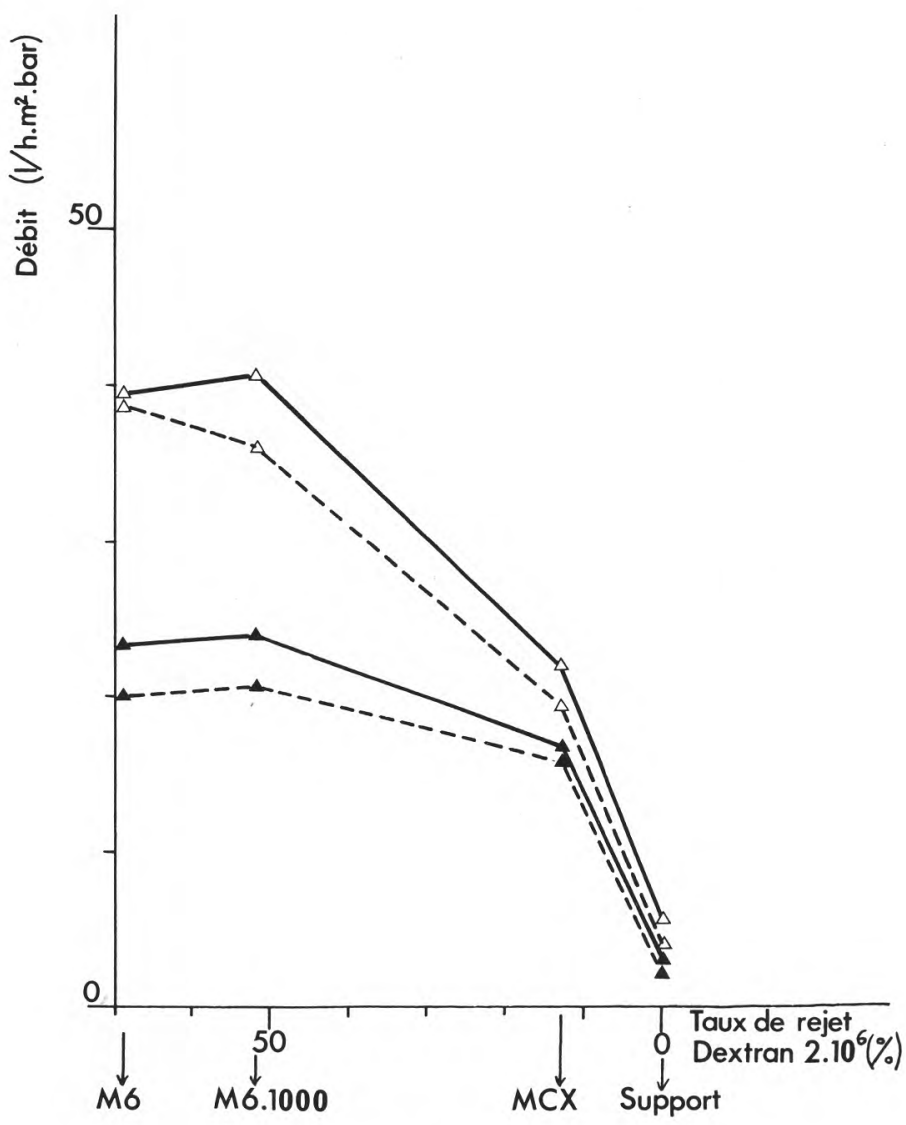

fig. 10

Débit de microfiltration après $1 \mathrm{~h} \mathrm{(-)}$ et $3 \mathrm{~h} \mathrm{(--)}$ pour des vitesses de balayage de $4 \mathrm{~m} / \mathrm{s}(\triangle)$ et $2 \mathrm{~m} / \mathrm{s}(\Delta)$ en fonction de la porosité de quatre types de membranes SFEC (porosité mesurée par le taux de rejet d'un Dextran de masse moléculaire $\left.2.10^{6}\right)$.

Microfiltration flux for flow speeds of $4 \mathrm{~m} / \mathrm{s}$ (-) and $2 \mathrm{~m} / \mathrm{s}$ (--) in function of porosity of four types of SFEC membranes (porosity was defined by rejection of Dextran having a molecular weight of 2.106 ).

fermées (M6 et M6 1000) pour lesquelles le débit est maximum et sensiblement égal dans les deux cas.

La diminution de la vitesse de balayage provoque un décroissement du débit surtout sensible pour les deux membranes M6 et M6 1000. 


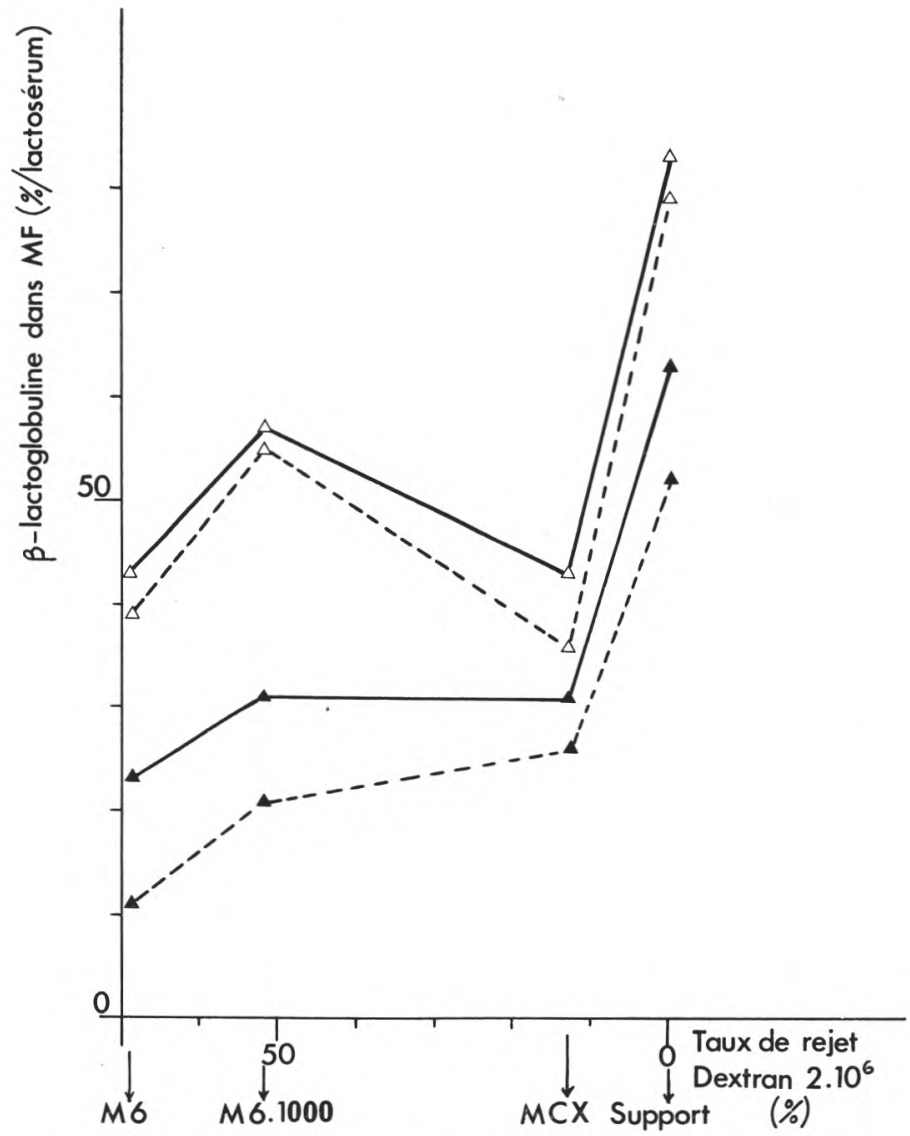

fig. 11

Pourcentage par rapport au lactosérum, de $\beta$-lactoglobuline dans le microfiltrat

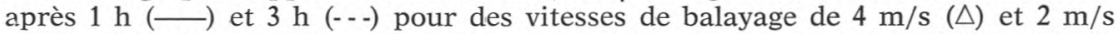
(A) en fonction de la porosité de quatre types de membranes SFEC exprimée comme indiqué figure 10 .

$\beta$-lactoglobulin permeation observed on SFEC membranes.

La perméabilité des protéines comparée au taux de rejet sur Dextran ne suit pas une courbe régulière. Cependant, la tendance semble être une augmentation irrégulière de cette caractéristique lorsque le taux de rejet sur Dextran diminue pour une vitesse de balayage de $2 \mathrm{~m} / \mathrm{s}$. A $4 \mathrm{~m} / \mathrm{s}$ les graphiques représentent plutôt une courbe en dents de scie. 


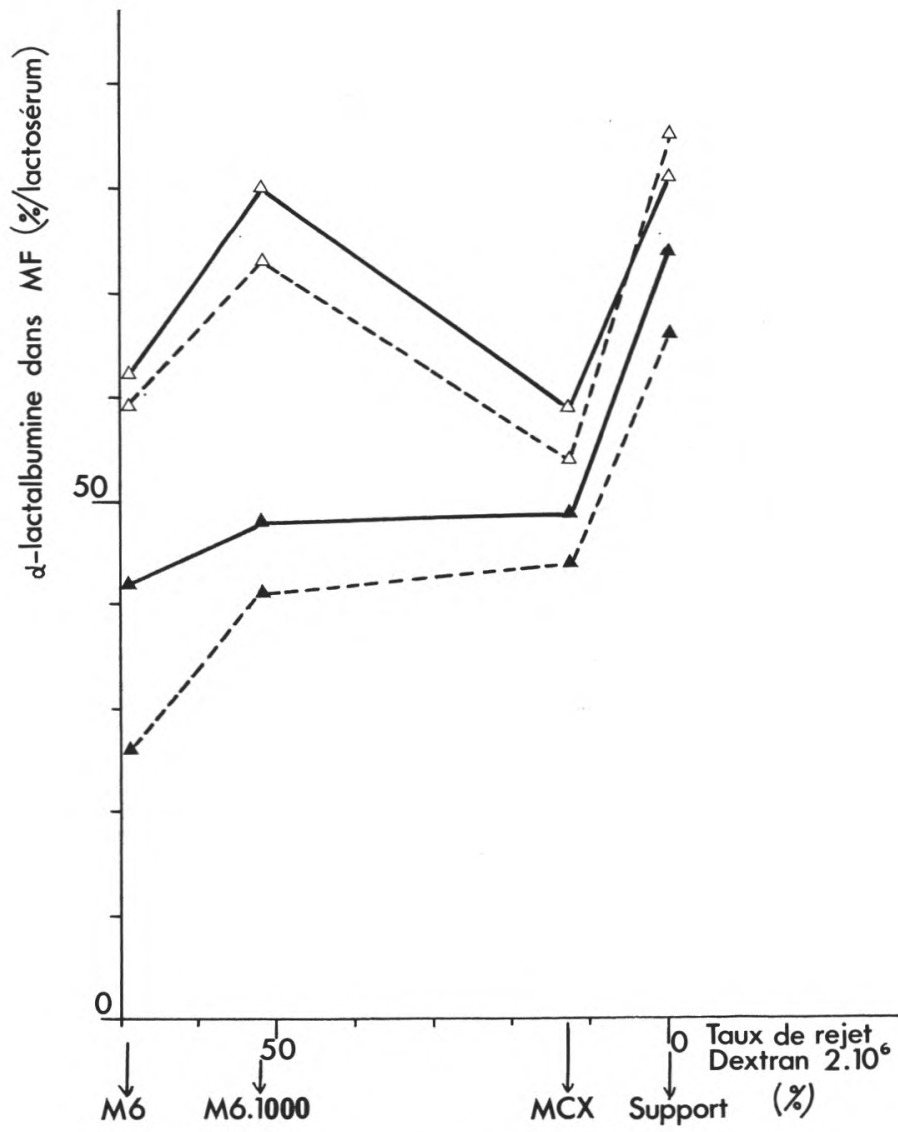

fig. 12

Pourcentage par rapport au lactosérum, d' $\alpha$-lactabulmine dans le microfiltrat après $1 \mathrm{~h}(-)$ et 3 heures $(--)$ pour des vitesses de balayage de $4 \mathrm{~m} / \mathrm{s}(\triangle)$ et $2 \mathrm{~m} / \mathrm{s}(\boldsymbol{\Delta})$ en fonction de la porosité de quatre types de membranes SFEC exprimée comme indiqué figure 10 .

ä-lactalbumin permeation observed with SFEC membranes.

Le passage des protéines varie dans le même sens que la vitesse de balayage.

L' $\alpha$-lactalbumine est moins retenue que la $\beta$-lactoglobuline.

Ces quatre types de membranes retiennent les bactéries de la flore totale dans des conditions satisfaisantes et retiennent totalement les bactéries coliformes. 


\section{CONCLUSION}

Aucune des membranes testées au cours de cette étude ne nous satisfait entièrement.

Le microfiltre idéal serait pour nous une membrane répondant aux critères suivants :

- rétention des bactéries lactiques permettant une réduction décimale de la flore du microfiltrat au moins égale à 3 ;

- rétention totale des bactéries coliformes;

- perméabilité vis-à-vis de la $\beta$-lactoglobuline et de l' $\alpha$-lactalbumine supérieure à $90 \%$;

- clarification du lactosérum;

- débit de microfiltration au moins égal à celui des membranes d'ultrafiltration.

Or, nous avons pu voir dans les chapitres précédents que seules les membranes Nuclépore et Sfec permettent une épuration bactérienne correcte mais le matériel Nuclépore est fragile et les membranes Sfec présentent soit une rétention trop importante des protéines du lactosérum, soit un débit trop faible dans les conditions actuellement utilisées. Quant aux membranes Rhône-Poulenc, elles ne remplissent qu'imparfaitement leur rôle d'écran bactérien.

Ces résultats sont donc relativement pessimistes mais nous ne désespérons pas d'améliorer les performances et la fiabilité des membranes de microfiltration dans un avenir proche. Cependant, un effort important devra être fait par les fournisseurs de matériel afin de nous permettre d'avancer rapidement. L'utilisation de microfiltrat de lactosérum pourrait être envisagée dans la préparation de concentrés de protéines très purifiés. Nous avons d'autre part pu vérifier au cours d'un essai de concentration d'un microfiltrat par ultrafiltration sur une installation Romicon équipée de membranes fibres creuses type PM 50 que le débit du perméat était supérieur de $30 \%$ à celui d'un lactosérum normal dans les mêmes conditions. Cette augmentation de débit peut être expliquée par l'absence de substances colmatantes (résidus de globules gras, lipoprotéines, fines de caséine...) au niveau de la couche de polarisation et aussi par le fait que cette absence entraîne également une diminution de la viscosité à concentration élevée. Les concentrés de protéines de lactosérum issus de lactosérums microfiltrés seraient une matière première de choix pour la protéinisation des boissons claires, la fragmentation en hydrolysats peptidiques en réacteur enzymatique à membrane, l'obtention de produits moussants, etc. 


\section{Bibliographie}

LeE (D. N.), MERson (R. L.) (1976). - Prefiltration of cottage cheese whey to reduce fouling of ultrafiltration membranes. J. Food Science, vol. 41, 403-410.

Merin (J.), GoRdin (S.), TANNy (G. B.) (1983). - Microfiltration of sweet cheese whey. New Zealand J. of Dairy Sc. and Technology, 18, 153-160.

ROGER (L.). - Contributions à la recherche d'une meilleure utilisation en alimentation humaine des composants glucidiques et protéiques du lactosérum grâce à l'emploi des techniques à membrane. Thèse E.N.S.A. Rennes (27-6-79). 